\title{
Hidden Violence:
}

\section{How COVID-19 School Closures}

Reduced the Reporting of Child

\section{Maltreatment}

Francisco Cabrera-Hernández, ${ }^{*}$ María PadillaRomo**
* Cabrera-Hernández is an Assistant Professor at University of Monterrey, Department of Economics, and a Research Fellow at the Center for Institutional Studies, Higher School of Economics, Moscow. Corresponding author: fcabrera@hse.ru.

** Padilla-Romo is an Assistant Professor at The University of Tennessee, Department of Economics. Contact at: mpadill3@utk. edu.

\begin{abstract}
This study examines how school closures during the COVID-19 pandemic affected the reporting of child maltreatment in Mexico City. We use a rich panel dataset on incident-level crime reports and victim characteristics and exploit the differential effects between school-age children and older individuals. While financial and mental distress due to the COVID-19 pandemic may result in additional cases of child maltreatment, synthetic control and difference-in-differences estimations document an average reduction in child maltreatment reports of $29 \%$ and $30 \%$, respectively, with larger reductions among females and in higher-poverty municipalities. These results highlight the important role education professionals in school settings play in the early detection and reporting of domestic violence against school-age children.
\end{abstract}

Keywords: Child maltreatment; Domestic Violence; COVID-19; School closures.

JEL classification: I29, I3 1, J12

\section{Introduction}

The proportion of children who report being victims of physical, sexual, or psychological violence is large. According to data from the Mexican Survey on Households' Dynamics (ENDIREH, 2016), 26\% of female teenagers aged 15-18 reported 
having been victims of maltreatment during childhood: $20.4 \%$ physical, $10.5 \%$ psychological, and 5.5\% sexual violence. Moreover, according to the National Survey on Children and Women (ENIM, 2015), 63\% of children between the ages of 1 and 14 were subjected to at least one form of psychological or physical punishment by members of their household, among whom $11 \%$ were subjected to severe physical punishment.

In addition to having short-term detrimental effects on children's health and well-being and, more broadly, the societal costs of law enforcement and providing care for its victims, violence on children has been shown to have long-lasting consequences. Victims of child maltreatment are more likely to commit a crime, be unemployed, and be welfare-dependent in adulthood (Zielinski, 2009; Gurrie \& Tekin, 2012). ${ }^{3}$ Evidence also shows that child maltreatment reduces victims' education and lifetime earnings (Currie \& Spatz Widom, 2010) in addition to exerting other negative spillovers. For example, Carrell, Hoekstra, \& Kuka (2018) show that being exposed to one peer victim of domestic violence in a class of 25 students during elementary school reduces earnings at ages $24-28$ by 3 percent.

As such, early detection is essential for mitigating the negative consequences of child maltreatment on both child development and on future economic outcomes. Yet, factors that drive abuse detection and reporting remain understudied. Recent evidence suggests that educators in school settings may play an important role in detecting and reporting cases of child maltreatment, since most children spend a large part of their day at school. For example, Fitzpatrick, Benson, \& Bondurant (2020) provide evidence that education professionals in the U.S. report cases that would have been otherwise missed, and thus, school closures may have caused under-detection and under-reporting of domestic violence.

In this paper, we contribute to the literature on domestic violence by estimating the effects of COVID-19 related school closures on the reporting of child maltreatment. We argue that the absence of education professionals had a detrimental effect on its detection and reporting. We do so by exploiting a rich panel of incident-level crime data - in particular, on domestic violence and child maltreatment - that comes from the reports of the Mexico City Attorney General's Office (Fiscalia General de Fusticia, FGJ). The data comprise information regarding the crime's location and date, and victim's gender and age, between January 2019 and June 2020. With this information, we build a weekly municipality-gender-age group panel which also includes information on people's mobility from Google's Global Position System (GPS) reports and information on municipalities' socioeconomic development to explore heterogeneous effects for relatively poorer and richer municipalities.

We leverage these data and exploit the March 23, 2020 Mexico City school shutdown in response to the COVID-19 pandemic as a natural experiment to estimate the impact of school closures on the reporting of school-age child maltreatment using synthetic control methods and a difference-in-differences research design. We first perform a synthetic control estimation of the average effect of school closures on reports of domestic violence perpetrated against schoolage children (aged 3 to 15). For this, we use the number of reports during the pre-treatment period for older individuals to build a synthetic control group. ${ }^{4}$

Second, in order to consider seasonal changes, municipality-level and gender-level differences and to disentangle different heterogeneous effects, we conduct a difference-in-differences strategy comparing weekly reports of domestic violence against school-age children within a given municipality before and after school closures, with respect to a comparison group com-

3 Child maltreatment is also associated with increased underage drinking and drug use (Vanderminden et al., 2019).

4 The donor pool contains older individuals who are grouped as follows: in five year gaps from 16 to 21 years old, 22 to 27 years old, up to the group aged 46-51. This way we have enough cases and variation per age group-gender, and by week-municipality. 
prised of individuals aged 16 to $21 .{ }^{5}$ Finally, we also estimate weekly event-study models to examine the plausibility of the parallel trends assumption and to explore treatment effect heterogeneity across time.

Most of the literature on reported crimes in developed and developing countries during the COVID-19 stay-at-home policies has focused on the effects on Intimate Partner Violence (IPV) and more specifically on reports of violence against women (Peterman et al., 2020). For example, findings for the U.S. suggest an increase between $10 \%$ and $27 \%$ on domestic violence reports (Boserup et al., 2020; Leslie \& Wilson, 2020), while, in developing countries, the literature suggests even larger impacts. Agüero (2020) uses data from the helpline for domestic violence in Peru and uses a differences-in-differences strategy. He finds that a one standard deviation decrease in a Google-based mobility index doubles the number of calls.

Similarly, Silverio-Murillo \& de la Miyar (2020) exploits the bans on alcohol consumption and the COVID-19 lockdowns across Mexico City to conduct an event-study analysis and explore their effects on the number of calls to "Línea Mujeres." While the authors do not find an overall effect on emergency calls, they report a 100\% increase in calls looking for psychological assistance. $^{7}$

However, child maltreatment in most of the cases can only be formally reported by an adult and not directly by the victim ${ }^{6}$ and in many cases, parents are the main perpetrators. Therefore, the potential effects of the pandemic on the detection and reporting of child maltreatment are not straight forward. On the one hand, the COVID-19 pandemic has the potential to increase violence against children because of the increased time at home and possible financial and psychological distress faced by parents. Indeed, previous research suggests that increases in domestic violence could be explained by increased exposure to perpetrators, and because of families' worsened overall economic conditions related to lower households' income, increases in unemployment, and mass-layoffs (Cunradi et al., 2011; Stephens-Davidowitz, 2013; Lindo et al., 2018).

On the other hand, the pandemic forced schools to close and so the reporting of child maltreatment by education professionals may have decreased or ceased. In this regard, Fitzpatrick, Benson, \& Bondurant (2020) using variation in discontinuities in school-entry laws and school calendars, provide evidence of an increase of 33 to 41 percent in the number of child maltreatment reports by education professionals. This is observed at the beginning and end of the academic year, as compared to the end and the beginning of summertime, when children are typically not in school. A natural question, then, is whether any potential increase in child maltreatment due to the pandemic may have been dampened by decreased reporting.

Our results from both synthetic control methods and difference-in-differences show an average reduction of $29 \%$ and $30 \%$ on the reporting of child maltreatment, or a reduction of 9 to 10 cases from the average of 33 per week. Further difference-in-differences estimations show that these effects are higher for females (a reduction of $66 \%$, or 22 cases per week from an average of 33) and in poorer municipalities (reduction of $40 \%$ or 14 cases less a week, from an average of 34) with no discernible effects on male children or in wealthier municipalities. The effects obtained with all methods are robust to different specifications and placebo estimations

5 We chose individuals 16 to 21 years old as a comparison group for three reasons: a) their potential to continue enrolled in formal education (approximately 50\% of them), while being more likely to fill a report on domestic violence themselves (i.e. $76 \%$ of the reports are filled by the victims vs. $1 \%$ in the case of school age children); b) the cumulative trend of domestic violence before school closures for this age group is similar to that for school-age children and c) after applying the synthetic controls strategy, the group of age 16-21 receives $96.8 \%$ of the weight among the age groups in the donor pool.

6 Linea Mujeres is a call service operated by Mexico City's government for reports of domestic violence.

7 Ravindran \& Shah (2020) applies a similar strategy on emergency calls in India and find an increase of $131 \%$ in domestic violence complaints in districts that faced the strictest lockdown measures. ${ }^{6}$ In our data this is the case in $99 \%$ of child maltreatment reports. 
and our event-study design offers evidence of similar pre-trends. In sum, these effects suggest that 220 incidents of child maltreatment for school-age females were not reported to the authorities in the 10 weeks after school closures. Moreover, this likely represents a lower bound as child maltreatment potentially increased during this period where abusers had more facetime with victims.

These findings contribute to the recent discussion regarding the effects of the COVID19 pandemic on domestic violence in developing countries, generally suggesting that the inability to meet financial obligations and maintaining social ties has significantly increased reported family stress and domestic violence Beland et al. (2020). However, we differentiate from existing work by focusing on the less explored topic of child maltreatment after stay-at-home policies were implemented. Moreover, we contribute to the debate on the importance of education professionals reporting this shadowed violence by exploiting the timing of school closures and by providing evidence from a broad set of models commonly used to examine natural shocks. We also differentiate from the previous literature by exploiting the year-to-year across groups of age comparison on reports classified by a judge as child maltreatment, rather than on calls to helplines that may involve a broader and more heterogeneous type of reports. Our contribution is important because while adult women in developing countries are deeply vulnerable, violence against children could be hard to detect given that they need of third-party adults to report.

Furthermore, our results of an overall reduction on the reporting of child maltreatment in Mexico City are supported by previous findings for the U.S. suggesting that poor economic conditions are associated to lower child maltreatment reports. For example, Stephens-Davidowitz (2013) shows that observed reductions during the 2008 Great recession were not due to actual decreases in maltreatment rates but rather large decreases in the reporting rates caused by the economic downturn. By providing evidence on alternative proxies for actual maltreatment rates, less likely to be affected by reporting rates, such as rates of child mortality from neglect and Google searches related to maltreatment, the author suggests that doubling unemployment increases child maltreatment from $10 \%$ to $24 \%$ but decreases reported child maltreatment incidents by $12.7 \%$. Therefore, our findings are more likely a proxy of a larger under-reporting for school-age children given school closures, rather than an actual fall in child maltreatment cases. In this regard, in Section 2, we also present descriptive evidence showing that emergency calls to report child maltreatment in Mexico City did not fall after school closures with respect to same weeks in 2019.

This paper directly complements research by Fitzpatrick et al. (2020) who exploit the school-entry laws and school calendars discontinuities to show education professionals are reporting cases that would have been missed otherwise. Our estimations are also in line with recent work by Baron, Goldstein, \& Wallace (2020) who test whether the number of child maltreatment allegations in March and April 2020 in Florida, are uncharacteristically high or low, relative to the number of allegations in the same months in other years of their sample considering seasonal and secular trends. The authors show that the actual number of reported allegations was approximately $27 \%$ lower than expected for these two months. However, we strengthen this evidence with the inclusion of a control group, and a richer set of fixed effects as controls that allow us to control for weekly seasonal effects and to exploit variations across municipalities and for different subgroups of the population in a developing country with high inequality.

The rest of the paper proceeds as follows. Section 2 presents the data we use as well as some descriptive figures. Section 3 explains our identification strategy. Section 4 presents the results. Section 5 draws conclusions. 


\section{Data}

We use information on domestic violence victims reported to the Mexico City Attorney General's Office (Fiscalia General de fusticia, FGJ) from January 2019 to June 2020. These data contain information on victim and crime characteristics. Specifically, they have information on gender, age, type of crime, and location and date of occurrence. We keep information on domestic violence reports and when they are reported and then aggregate these data weekly at the municipality-age group-gender level. ${ }^{8}$

We define child maltreatment as domestic violence against school-age children (3 to 15 years). While we do not have information on the specific type of maltreatment, it is important to note that civil judges are the ones in charge of classifying the crime reports as domestic violence, based on legal criteria, and so making the overall group of crimes considered as domestic violence on children, rather homogeneous. We also create 6 age groups of older individuals by 5-year gaps in age: 16-21, 22-27 and so forth up to group 46-51 years old. This way we maintain enough data and sufficient variation across time and within age groups. We combine these data with population counts by municipality-age group-gender from Mexico's 2015 Intercensal Survey.

We also use GPS (Global Position System) data reported by Google for Mexico City, showing changes in mobility compared to a baseline of 10, set by Google on February 15, 2020. These data include mobility changes in retail, pharmacy and grocery shopping, public transportation, recreation, and visits to public spaces, as well as mobility in workplaces. For presentation purposes, we build a mobility index averaging all categories, ranging from zero (low mobility) to 10 (high mobility).

Additionally, we use the Social Development Index (SDI) calculated by Mexico City's Council for the Evaluation of Social Development (Evalua, in Spanish) to identify the municipalities with the highest socioeconomic development or in the top quartile. ${ }^{9}$ This index gathers information on households' infrastructure conditions, access to dwelling, access to education, sanitation infrastructure, and access to electricity. ${ }^{10}$ In our panel, $24 \%$ of reports of child maltreatment are concentrated in counties with a high SDI while $75 \%$ of the victims across all age groups are females. ${ }^{11}$

Figure 1 shows the 7-day moving average trends of daily domestic violence reports, 30 days before and 70 days after the COVID-19 pandemic-related preschool, elementary, and middle school closures on March 23, 2020, when a state-mandated stay-at-home order started, and for the same period in 2019. ${ }^{12}$ We show trends for (a) school-age children (3-15) and for the three age groups immediately following school-age (b) individuals aged 16-21 (c) 22-27 and (d) 28-33. Note that, while reports of domestic violence started to fall for all groups of age from ten days before school closures and with respect to 2019, Panel (a) shows that the weekly average reports for school-age children sharply decreased after the schools closed, in comparison with their own tendency in 2019 and with respect to older individuals in panels (b)-(d). We argue that mobility restrictions and stay-at-home orders affected the report of domestic violence for all individuals, but school-age children trends are disproportionately reduced just after school closures. This is key for our identification strategy as we detail in Section 3.

8 Mexico City has 16 local councils or "Alcaldías" that act as local sub-regional governments with their own socioeconomic and political characteristics. Formally, they act similarly to municipal governments.

9 This index is published in Mexico City's official gazette on March 4, 2016 and can be found in: https://evalua.cdmx.gob.mx.

10 The top quartile identifies four municipalities as the most advantaged: Cuauhtémoc, Miguel Hidalgo, Coyoacán, and Benito Juárez. These municipalities have historically been known for having good living standards, good access to services and lower crime rates.

11 Proportions by age group can be consulted in the Annex Table A1.

12 The 7 -day moving average is $(3,1,3)$ or 3 days before the date, the actual date, and three days after 
Figure 1. Domestic Violence Reports by Age Group: 2019-2020

(a) School-Age (3-15) Children

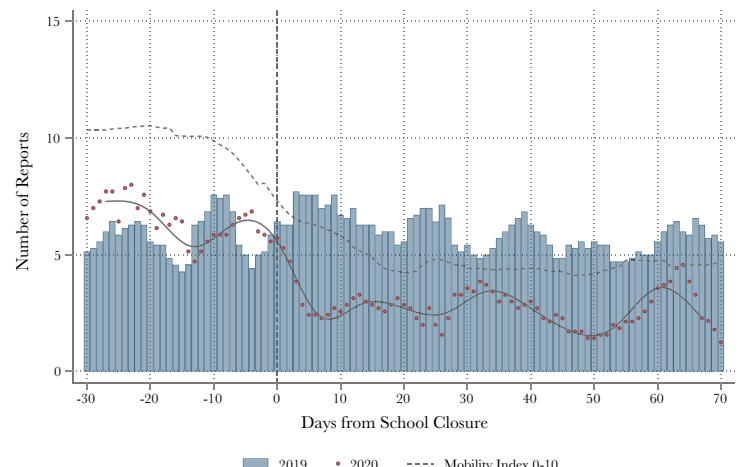

(c) Age 22-27

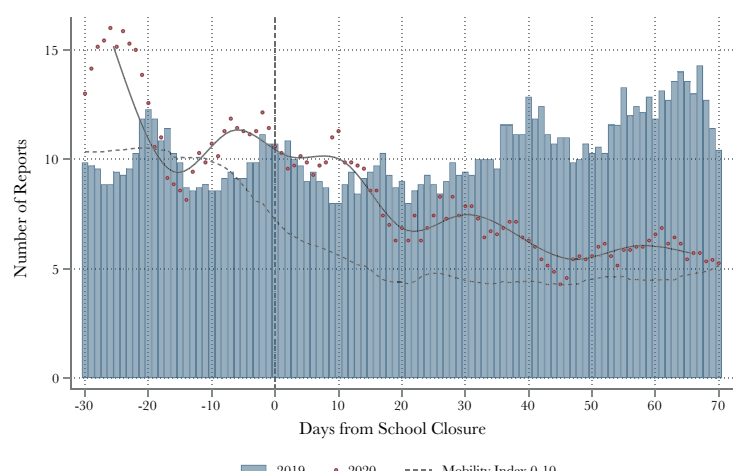

(b) Age 16-21

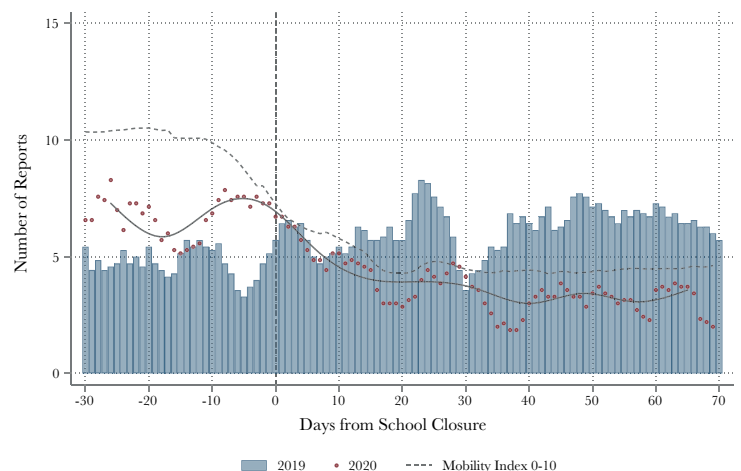

(d) Age 28-33

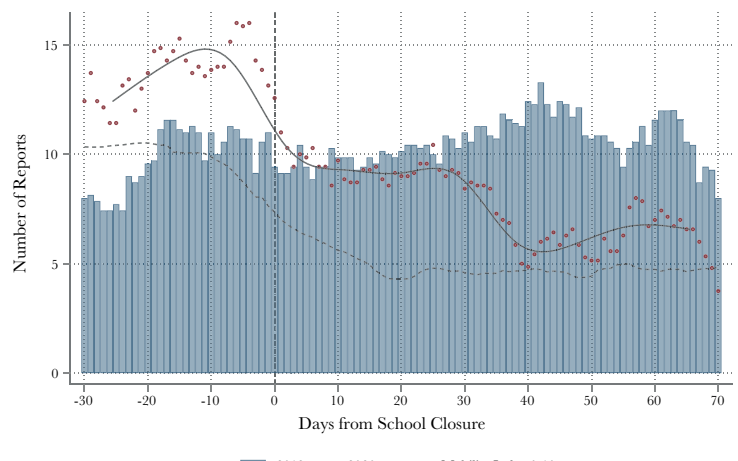

$2019 \quad \cdot 2020 \quad \cdots$ Mobility Index 0.10

Notes: Each panel shows the 7-day moving average $(3,1,3)$ of domestic violence reports for each age group and for the mobility index, with a base of 10 that is set on February 15, 2020.

The general fall in reports of domestic violence comes despite the criminal justice system being deemed an essential sector to continue operating during the COVID-19 related closures. In the case of older age groups (16 or older), before and after school closures, this reduction seems to relate more to the overall change in mobility across the city, which started to fall on March 13, 2020, when GPS tracking suggests people started to spend more time at home. This is 10-days before school closures and state-mandated social distancing, along with the closure of non-essential economic sectors. However, it is worth noting that there is no discontinuity coinciding with the school closures in the reporting trends of older age groups. Finally, reports of domestic violence during this period and for all age groups never returned to their pre-social distancing/ school closures levels, nor the trends observed in 2019, suggesting that general under-reporting of domestic violence will potentially last while social distancing prevails.

Finally, Figure 2 shows the number of emergency calls made by women, to report child maltreatment (against individuals younger than 18 years old). ${ }^{13}$ This includes the 911 emergency line and "Línea Mujeres," a domestic violence hotline operated by Mexico City's government. Note that after school closures, call volume does not fall with respect to the previous year, suggesting that the violence reported from home, as opposed to through teachers or school officials, remained constant. This contrasts with the fall for school-age children in cases reported to the authorities.

13 The dataset only contains information regarding the age of the caller, but it does not contain details on the age of the victim, for which we cannot separate by age of the victim. 
Figure 2. 911 and "Línea Mujeres" Calls on Child Maltreatment: 2019-2020

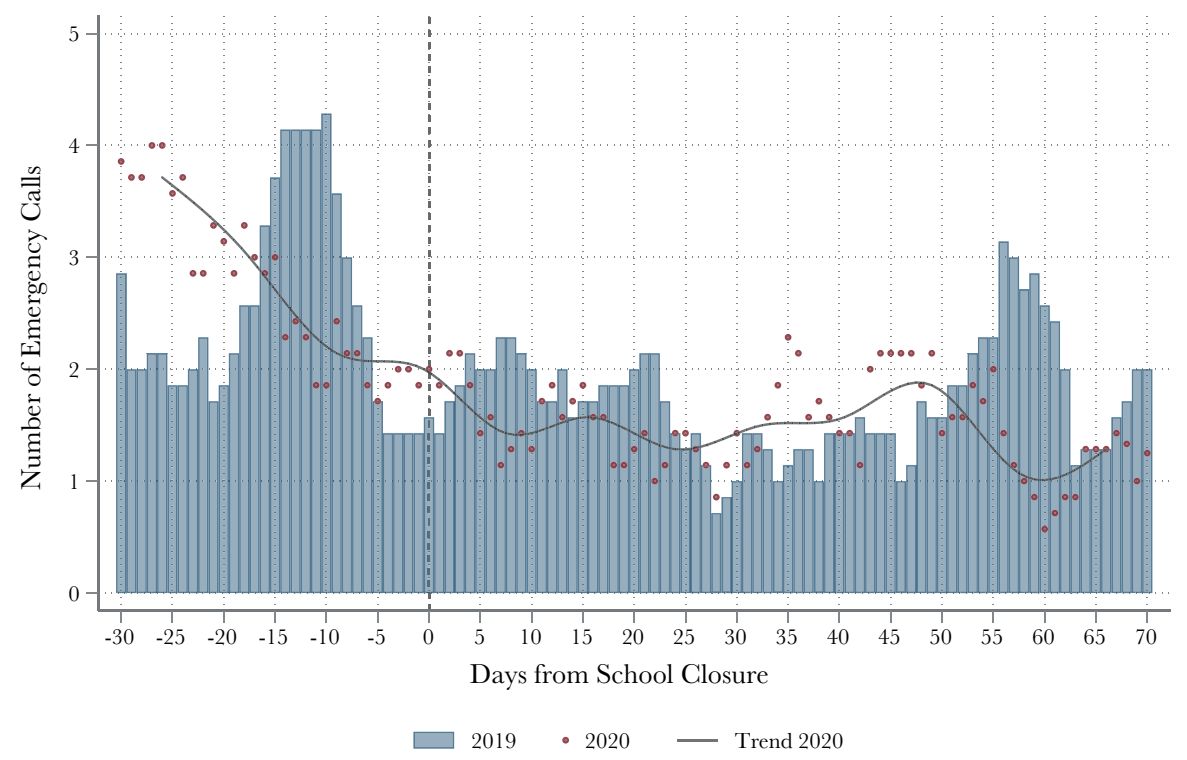

Notes: This figure shows the 7-day moving average (3,1,3) of Mexico City's 911 and "Línea Mujeres" emergency calls related to domestic violence when the victim is a minor (aged 0 to 17 years).

\section{Empirical Strategy}

We exploit the March 23, 2020 Mexico City school shutdown as a natural experiment and estimate the impact of school closures on the reporting of child maltreatment using synthetic control methods and a difference-in-differences research design.

Note that if we compare reports of child maltreatment before and after school closures, we would not consider seasonal changes and teachers' potential involvement. To account for this possibility and for municipality level differences within Mexico City, in our difference-in-differences setting, we compare weekly reports of domestic violence perpetrated against school-age children (age 3 to 15) within a given municipality before and after schools closed, relative to the same measure in the previous year (2019), and with respect to a comparison group comprised of individuals aged 16 to 21 years. We argue that while the stay-at-home orders reduced the reporting on domestic violence, school-age-children were disproportionately affected by the school closures.

We chose individuals aged 16 to 21 years as a comparison group for three reasons. First, like their treatment group counterparts, they are likely to attend school but are less likely to dependent on third parties to file domestic violence reports on their behalf. ${ }^{14}$ Second, the $16-21$ yearold group saw similar trends in reporting prior to the school closure date. Third, the 16-21-yearold age group made up just over 96 percent of the synthetic counterfactual.

Hence, we first use the synthetic control method introduced by Abadie, Diamond, \& Hainmueller (2010) to estimate the effects of school closures on child maltreatment reports.

This method uses pre-intervention data to create a [synthetic] control group similar in outcomes to the treatment group. We use all six age groups as potential donors and estimate the average effect on the treated group of school-age children. That is, the estimate for the [synthetic] counterfactual for child maltreatment reports is $\Sigma_{j} \omega_{j} Y_{j w y}$, where $\omega_{j}$ is the weight assigned to the donor age group $j$ and is calculated by minimizing the distance between the observed number

14 In our data, 99\% of the domestic violence reports for school-age children are filled by a third party, while for the group of age 16 to $21,76 \%$ of the reports are filled by the victim and $24 \%$ by a third party. 
of child maltreatment reports $\left(Y_{i}\right)$ and $\Sigma_{j} \omega_{j} Y_{j w y}$ for all pre-treatment periods. We also present placebo graphical evidence assigning all control age groups as "treated."

We then focus on the difference-in-differences setup comparing school-age children to the group of individuals aged 16-21 years. We define weeks as Monday to Sunday such that school closures relate to the same week (13) in both years, 2019 and 2020, to be able to control for seasonal changes. Additionally, to consider the count nature of our child maltreatment data and the large number of zeroes, we estimate a fixed effects Poisson regression model by Pseudo Maximum Likelihood (PPML). ${ }^{15}$ Specifically, we rely on Correia, Guimaraes, \& Zylkin (2020)'s addition of the ppmlhdfe command to Stata, which allows the inclusion of multiple high-dimensional fixed effects. Our Poisson regression model is given by:

$$
\text { Maltreatment }_{m g w y}=\exp \left[\alpha_{m}+\gamma_{w y}+\theta \text { SchoolAge } e_{g}+\delta \text { SchoolAge }{ }_{a} \times \text { Closure }_{w y}\right] u_{m g w y}
$$

where Maltreatment ${ }_{m g w y}$ is the number of domestic violence reports in municipality $m$, for age group $g$, in week-of-the-year $w$ in year $y ; \alpha_{m}$ are municipality fixed effects that allow us to control for time-invariant differences between municipalities; $\gamma_{w y}$ are week-by-year fixed effects that control for secular trends of domestic violence. SchoolAge is an indicator variable that equals one for school-age children (3-15 years), and zero otherwise;

Closure $_{w y}$ is an indicator variable that equals one after schools closed in week 13 of year 2020, and zero otherwise; and $u_{m g w y}$ is an error term which we allow to be correlated within municipalities-by-gender. We also include an exposure variable in our model - the population in each municipality-age group-gender combination - to allow the counts of domestic violence reports to be comparable across municipalities. The coefficient of interest is $\delta$ and it measures the effects of school closures on child maltreatment reports.

Additionally, to consider potential variation across municipalities and week-years which could result in different levels of domestic violence and seasonal trends, in our preferred specification we include a set of fixed effects as controls, including gender of the victim and municipality-specific year and week-of-the-year fixed effects. Municipality-by-year fixed effects aim to control for within-municipality differences in reports of domestic violence from one year to the other; municipality-by-week fixed effects would control for municipality-specific seasonal trends. Finally, to capture first-order trends in the number of reports within each municipality and within each gender, in some specifications, we include municipality-specific and gender-specific linear time trends.

For $\delta$ to have a causal interpretation, we must assume that reporting trends would not differ between school-age children and older victims of domestic violence absent school closures. We provide graphical and empirical evidence supporting this assumption. In Figure 3, we show the cumulative number of reports across days in 2020 for school-age children and for age group 16 to 21 . The reporting gap between school-age children and older individuals remains stable before schools closed. The trends for all groups begin to diverge after school closures, but the reduction in growth seems stronger for school-age children compared to individuals aged 16 to 21, suggesting that school closures affected more the reporting of violence for school-age children. We also provide evidence of no differences in pre-treatment trends in an event-study setting. However, we note that it is possible that maltreatment increased after schools' shutdown and thus, and regardless of the method, our estimates can be considered as a lower bound of the true effects. 
Figure 3. Cumulative Number of Domestic Violence Reports by Age Group

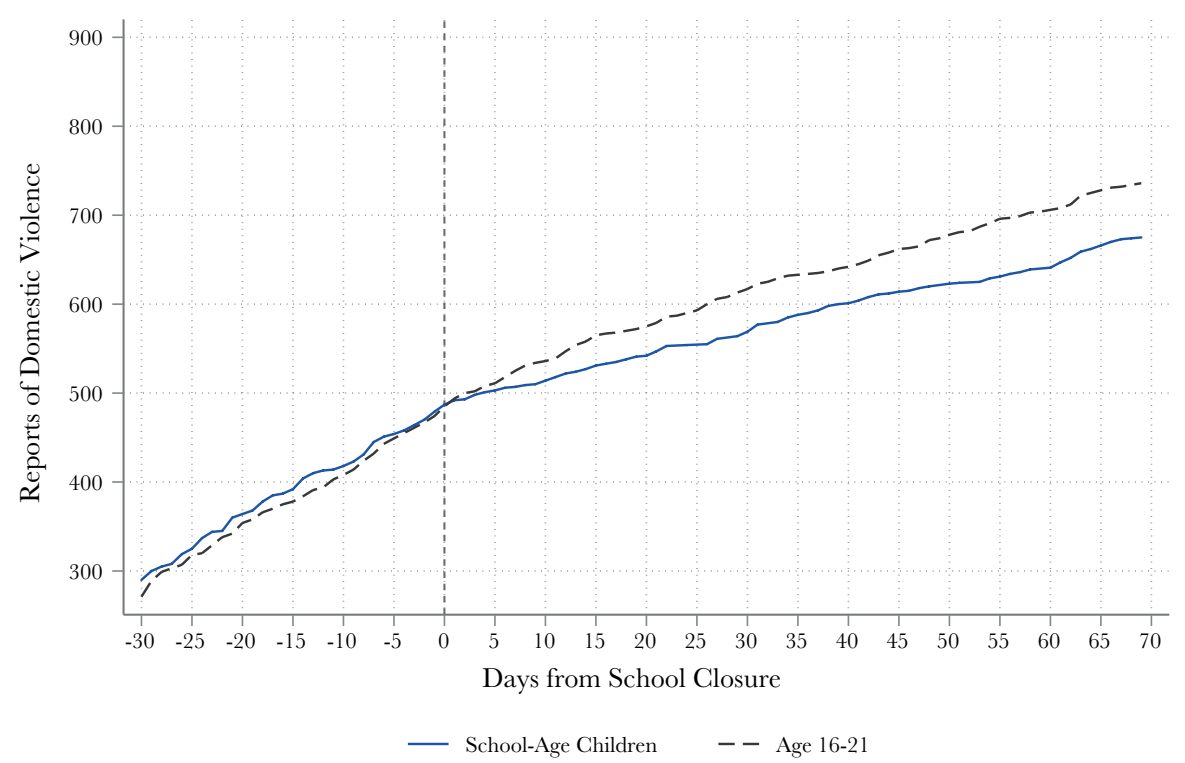

Notes: This figure shows the cumulative number of domestic violence reports 30 days before and 70 days after schools closed separately for school-age children and for individuals aged 16-21 years.

Finally, we estimate weekly event-study models to examine the plausibility of the parallel trends assumption and to explore treatment effect heterogeneity across time. The estimated effects are relative to the differences in maltreatment reported seven or more weeks before school closed. The models also include the set of dummies in Equation 1 that include municipality seasonal and secular trends, making this specification a within-municipality comparison of weekly reports in 2020 relative to 2019 and to the age group 16-21 years.

\section{Results}

To motivate the use of our difference-in-differences research design, we begin the analysis by showing the estimated effects of school closures on child maltreatment reports using a synthetic control method, which allows us to identify a data-driven counterfactual and to shed light on the size and robustness of our results. We then use age group 16-21 as a control group in a difference-in-differences framework and explore treatment effect heterogeneity across time, gender, municipalities' social development, and age. ${ }^{16}$

\section{Synthetic Control Method}

Panel (a) in Figure 4 shows the trends of the log of weekly domestic violence reports for schoolage children and its synthetic counterpart. The matching was based on pre-treatment outcomes (before week 65, where week 1 is January 1, 2019). The predictors are the observed log of number of domestic violence reports for all age groups before school closures. The synthetic control group is comprised of age groups 16-21 (96.8\%), 46-51 years (2.5\%) and all other age groups $(0.7 \%)$. The differences presented in Panel (b) imply a weekly reduction of $29 \%$. We also calculated a permutation-based p-value $(<0.001)$ that uses the ranking of school-age children's reports relative to the placebo age groups after school closures. ${ }^{17}$

16 That is, preschool, elementary, or middle school age children.

17 That is, after assigning treatment to all the other (control) groups of age and comparing these to an estimated synthetic control for each case, no other comparison showed a reduction in reports at least as big as the one presented for school-age children. 
Figure 4. Estimated Effects of School Closures on Child Maltreatment Reports

(a) Synthetic Control Trends

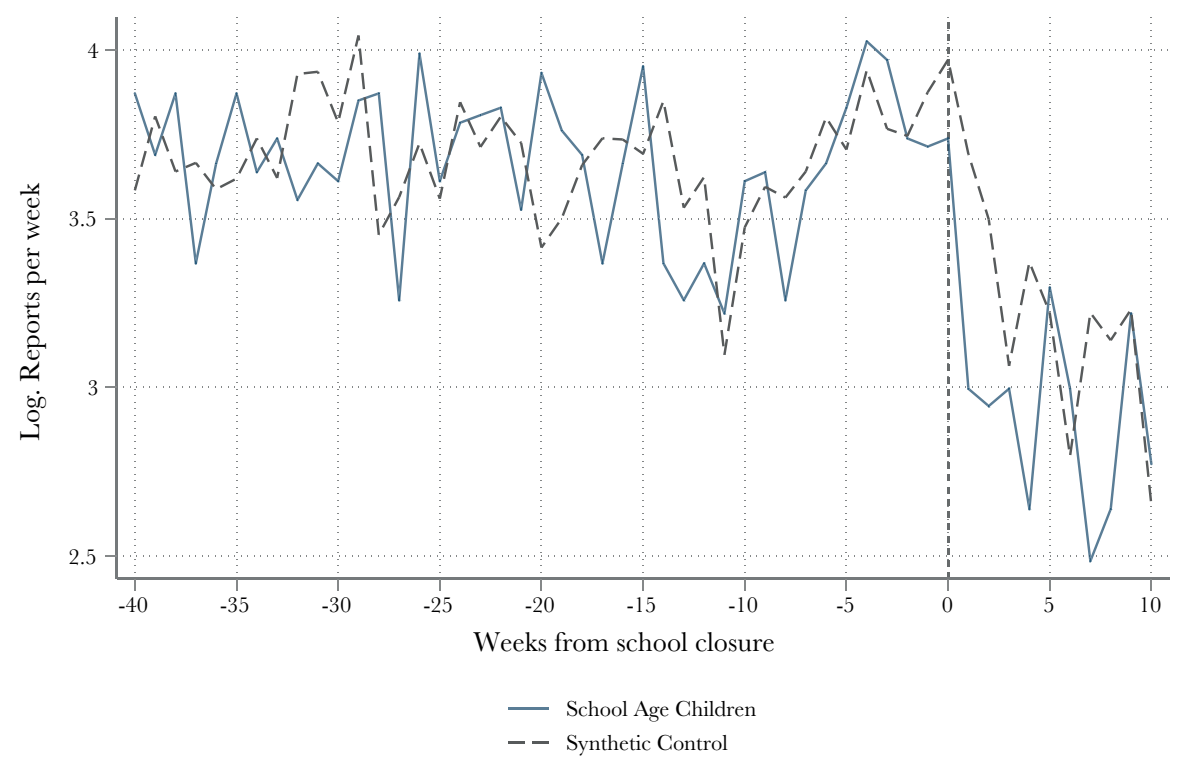

(b) Synthetic Control Differences

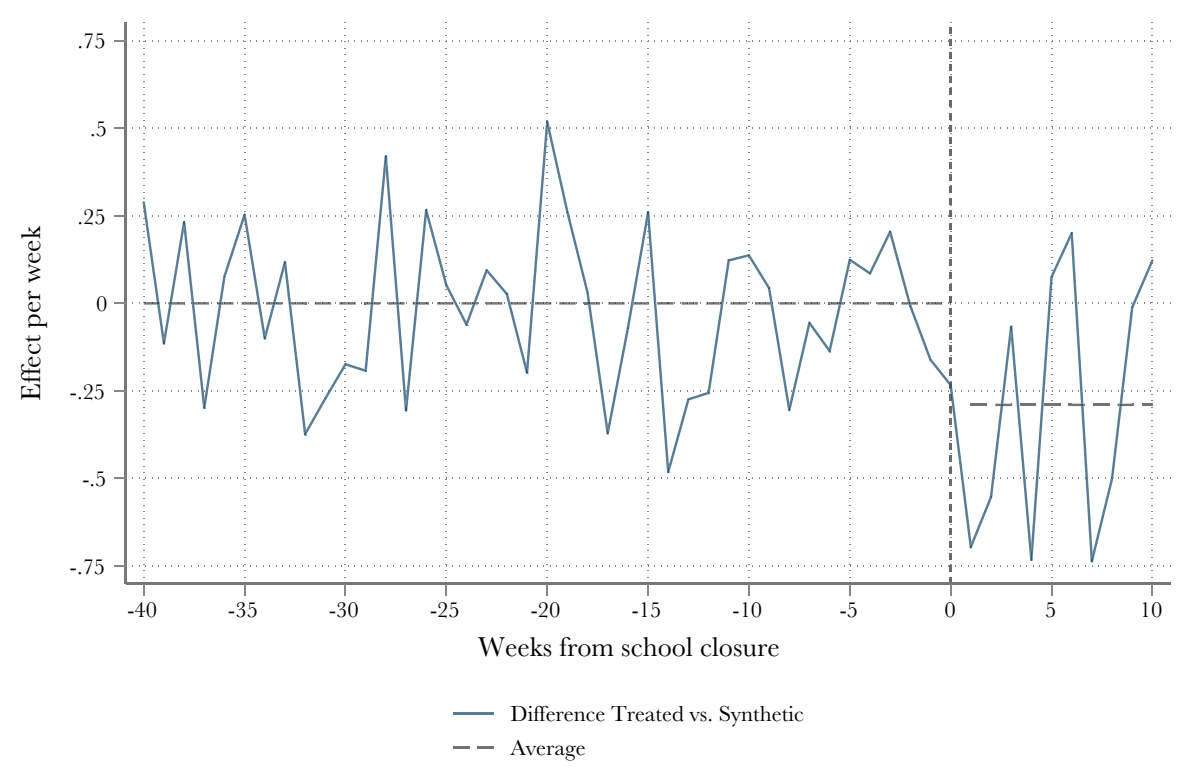

Notes: Estimated effects are generated using synthetic control methods. Synthetic control weights are calculated based on pre-treatment (before week 65, where week 1 is set on January 1, 2019) log of domestic violence reports for all age groups. The synthetic control group is comprised of age groups 16-21 (96.8\%), 4651 years $(2.5 \%)$ and all other age groups $(0.7 \%)$. Permutation based $p$-value 0.001 . The differences presented in Panel (b) imply an average reduction of $29 \%$ reports per week.

To support the main findings from the synthetic control approach, Figure 5 shows a series of estimated effects as if all age groups in the donor pool were treated or affected by school closures. The gray lines show the difference in logs of domestic violence reports between each age group in the donor pool and its respective synthetic counterpart while the blue line shows the difference between school age children and its synthetic version. The results indicate that during the first few weeks after school closed, the estimated effect for school-age children is unusually large relative to the distribution of changes after treatment for the other age groups in the donor pool. 
Figure 5. Placebo Synthetic Controls

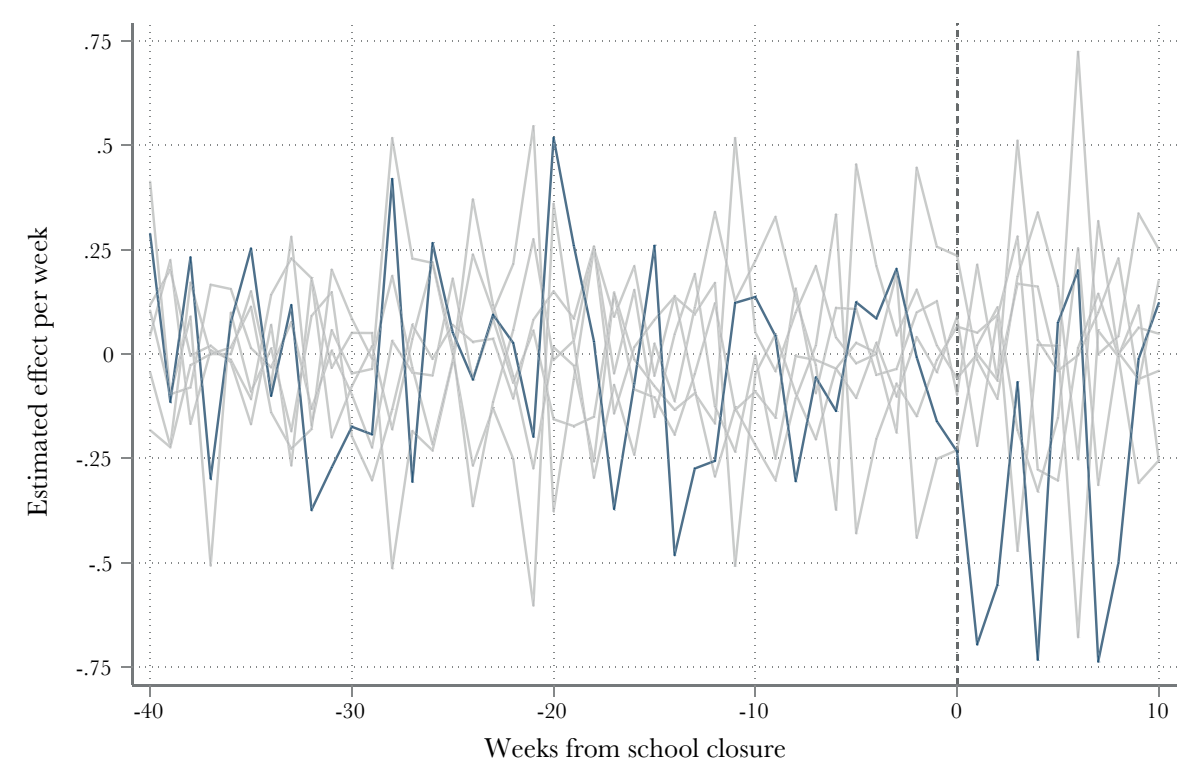

Notes: Estimated effects are generated using synthetic control methods. Synthetic control weights are calculated based on pre-treatment (before week 65, where week 1 is set on January 1, 2019) log of domestic violence reports for all age groups. The synthetic control group is comprised of age groups 16-21 (96.8\%), 46-51 years (2.5\%) and all other groups $(0.7 \%)$.

\section{Difference-in-Differences}

Table 1 shows the estimated effects of school closures on child maltreatment reports based on our baseline specification in Equation 1. The estimated effects can be interpreted as the weekly percent change in the number of domestic violence reports. Column 1 presents the baseline specification, which includes gender, school-age, municipality, and week-year fixed effects. In columns 2 and 3, we also allow seasonal and year trends to vary across municipalities by including municipality-by-year and municipality-by-week fixed effects. Finally, in columns 4 and 5, respectively, we include municipality-specific and gender-specific time trends. Regardless of the specification, the estimated effects indicate an average reduction in child maltreatment reports of 30 percent after school closures. Note that even when we relax the parallel trends assumption by including municipality-specific and gender-specific linear time trends the estimated effects remain unchanged, supporting our identification strategy.

Table 1. Estimated Effects on Weekly Reports of Domestic Violence on School Age Children

\begin{tabular}{lccccc}
\hline & $(1)$ & $(2)$ & $(3)$ & $(4)$ & $(5)$ \\
\hline School Age X Glosure & $-0.301^{* * * *}$ & $-0.303^{* * *}$ & $-0.305^{* * * *}$ & $-0.305^{* * * *}$ & $-0.305^{* * *}$ \\
& $(0.106)$ & $(0.105)$ & $(0.105)$ & $(0.105)$ & $(0.105)$ \\
R-squared & & & & & \\
N & 4736 & 4736 & 4612 & 4612 & 4612 \\
Municipality-by-year & No & Yes & Yes & Yes & Yes \\
Municipality-by-week & No & No & Yes & Yes & Yes \\
Municipality-specific time trends & No & No & No & Yes & Yes \\
Gender-specific time trends & No & No & No & No & Yes \\
\hline
\end{tabular}

Notes: Each column represents a different regression. All estimations include municipality, school age, gender, and week-year fixed effects. Standard errors in parentheses are clustered at the municipality-by-gender level.

Estimates are relative to age group 16-21.

$*, * *, * * *$ Significant at the $10 \%, 5 \%$, and $1 \%$ levels, respectively. 
We also address the plausibility of the parallel trends assumption and explore treatment effect heterogeneity across time by using an event-study type analysis. Specifically, using our preferred specification in Table 1 Column 5, we interact our treatment variable with a set of dummies indicating the weeks prior to $(-6$ to -1$)$ and after school closures (0 to 9), so that all estimates are relative to the differences seven or more weeks before school closed. The estimated effects are presented in Figure 6. We observe flat, close to zero, and statistically insignificant effects in the weeks before school closures. Note that, while the reports seem to start declining two weeks prior to the official shutdown, when, according to Google's mobility index people started to spend more time at home; these effects (-2 and -1 weeks from school closures) are not statistically significant at conventional levels. The estimated effects on the weeks after schools closed are mostly negative and statistically significant, especially in the first few weeks after schools closed. Together, these results support our difference-in-differences identification strategy and show some heterogeneous effects across time.

Figure 6. Event-Study Estimated Effects of School Closures on Child Maltreatment Reports

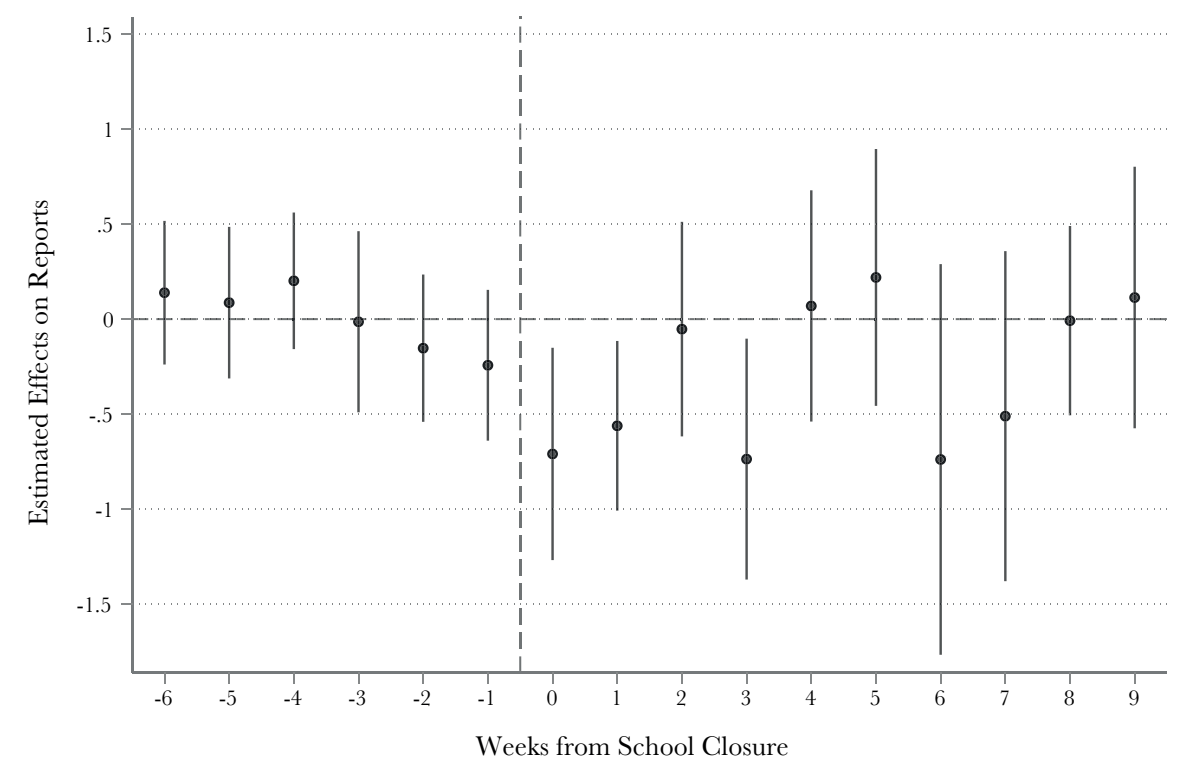

Notes: All coefficients and their 95\% confidence intervals come from an event-study specification that includes municipality, week-year, school age, gender, municipality-year, and municipality-week fixed effects as well as municipality-specific and gender-specific time trends. All estimates are relative to the difference in the number of reports seven or more weeks prior to school closures. Standard errors are clustered at the municipality-by-gender level.

We provide further evidence favoring the validity of our difference-in-differences findings by conducting the same set of regressions on older individuals that should not be affected by school closures. In other words, if the reduction on the reports of domestic violence for school-age children is not related to teachers and school officials' actions and relates instead to other unobserved factors not captured by our model, the same reductions could potentially be observed for other age groups. Panel A in Table 2 show the same specifications presented in Table 1, but the treatment is assigned to the group aged 22 to 27 (compared to the same control group 16 to 21). Note that, regardless of the specification, all the coefficients are not statistically significant. Panel B shows the same for the group of age 28 to 33 showing an effect with the opposite sign and significant only at the $10 \%$ level, offering support to our main findings. 
Table 2. Estimated Effects on Weekly Reports of Domestic Violence on Older Individuals

\begin{tabular}{lccccc}
\hline & $(1)$ & $(2)$ & $(3)$ & $(4)$ & $(5)$ \\
\hline Panel A. Effects on Group of Age 22-27 & 0.096 & 0.099 & 0.103 & 0.103 & 0.103 \\
Age 22-27 X Closure & & & & & \\
& $(0.065)$ & $(0.066)$ & $(0.067)$ & $(0.067)$ & $(0.067)$ \\
N & 4736 & 4736 & 4676 & 4676 & 4676 \\
Panel B. Effects on Group of Age 28-33 & & & & & \\
Age 28-33 X Closure & $0.151^{*}$ & $0.153^{*}$ & $0.151^{*}$ & $0.151^{*}$ & $0.151^{*}$ \\
& & & & & \\
N & $(0.088)$ & $(0.089)$ & $(0.090)$ & $(0.090)$ & $(0.090)$ \\
Municipality-by-year & 4736 & 4736 & 4700 & 4700 & 4700 \\
Municipality-by-week & No & Yes & Yes & Yes & Yes \\
Municipality-specific time trends & No & No & Yes & Yes & Yes \\
Gender-specific time trends & No & No & No & Yes & Yes \\
\hline
\end{tabular}

Notes: Each column in each panel represents a different regression. All estimations include municipality, weekyear, school age, gender, municipality-year, and municipality-week fixed effects as well as municipality-specific and gender-specific time trends. Standard errors in parentheses are clustered at the municipality-by-gender level. Estimates are relative to age group 16-21.

*, **, *** Significant at the $10 \%, 5 \%$, and $1 \%$ levels, respectively.

\section{Treatment Effect Heterogeneity}

In recent years, Mexico has seen a surge in female violence to the extent that, according to the UN-Women, 10 females are murdered every day across the country. Systemic violence against women may well have its roots at home and from early ages and this could have been exacerbated by stay-at-home policies. Similarly, one can think that lower socioeconomic sectors, with higher levels of informality and less access to social protection, could be more vulnerable to the economic shocks caused by the GOVID-19 pandemic. Consequently, economically disadvantaged people could suffer higher psychological and economic distress that could increase general levels of violence, including child maltreatment.

In this section, we explore treatment effect heterogeneity by victims' gender and by municipalities' socioeconomic conditions. In particular, we estimate the effects of school closures on child maltreatment reports separately for males and for females and for individuals in poorer (bottom three quartiles of the Social Development Index) and richer municipalities (top quartile), using our preferred specification in Table 1 Column 5 and interacting the variable of interest with a set of dummies that allow for different slopes for males and females and for relatively richer and poorer municipalities. The estimated effects are shown in Table 3. Column 1 presents the overall effect. In columns 2 and 3, we show the estimated effects, respectively, for females and for males. Finally, in columns 4 and 5, we present the estimated effects for poorer and richer municipalities, respectively. The results indicate a significantly larger reduction on the reporting of domestic violence for school-age females of 67 percent and of 42 percent in poorer environments with no discernible effects on males nor on relatively richer municipalities. This statistical differences in the effects between groups can be visually contrasted in Figure 7. 
Table 3. Estimated Effects by Gender and Municipalities' Social Development Index
(1) Overall
(2) Female
(3) Male
(4) Poorer
(5) Richer

\begin{tabular}{|c|c|c|c|c|c|}
\hline \multirow[t]{2}{*}{ School Age X Closure } & $-0.305^{* * *}$ & $-0.668 * * *$ & 0.214 & $-0.419 * * *$ & 0.111 \\
\hline & $(0.105)$ & $(0.130)$ & $(0.172)$ & $(0.138)$ & $(0.300)$ \\
\hline \multicolumn{6}{|l|}{ R-squared } \\
\hline $\mathrm{N}$ & 4612 & 4612 & 4612 & 4612 & 4612 \\
\hline Municipality-by-year & Yes & Yes & Yes & Yes & Yes \\
\hline Municipality-by-week & Yes & Yes & Yes & Yes & Yes \\
\hline $\begin{array}{l}\text { Municipality-specific time } \\
\text { trends }\end{array}$ & Yes & Yes & Yes & Yes & Yes \\
\hline Gender-specific time trends & Yes & Yes & Yes & Yes & Yes \\
\hline
\end{tabular}

Notes: Each column represents a different regression. All estimations include municipality, gender, week-year, municipality-week, and municipality-year fixed effects as well as municipality-specific and gender-specific linear time trends. Standard errors in parentheses are clustered at the municipality-by-gender level.

$*, * *, * * *$ Significant at the $10 \%, 5 \%$, and $1 \%$ levels, respectively.

Figure 7. Heterogeneous Effects of COVID-19 School Closures on Child Maltreatment by Gender and Municipalities' Social Development Index

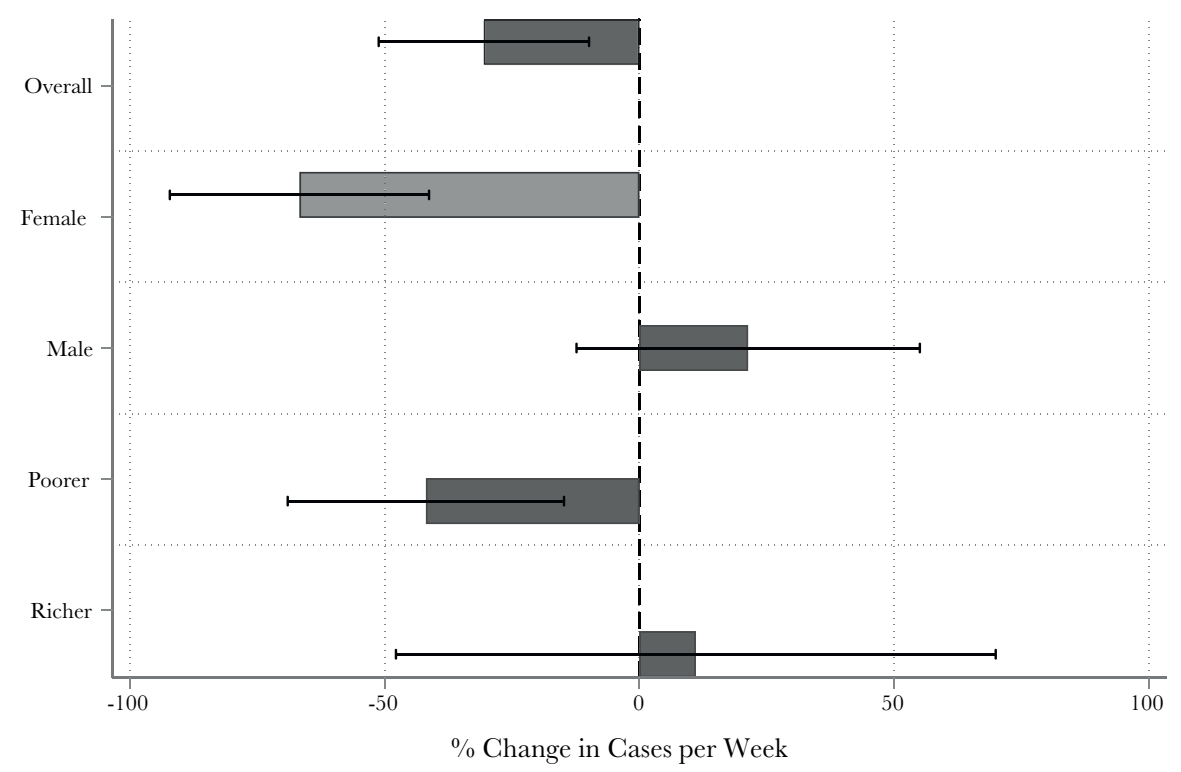

Notes: Each bar represents a different regression. All estimations include municipality, gender, week-year, municipality-week, and municipality-year fixed effects as well as municipality-specific and gender-specific linear time trends. Standard errors for 95\% confidence intervals are clustered at the municipality-by-gender level.

We further investigate heterogeneity by separating our sample of school-age children into three groups: preschool (aged 3-5), elementary school (aged 6-12), and middle school (aged 1315). We also use these results to argue the importance of teachers and school professionals in the detection and reporting of child maltreatment. The intuition is that while all basic education is mandatory by law, only elementary school coverage is universal (children aged 6-12). For example, approximately $30 \%$ of children aged 3-5 are not enrolled in a preschool, and thus, their reporting should not vary (or at least not as much as in elementary school) given that a good proportion of these children is not in contact with teachers or other school staff.

The estimated effects for these groups are shown in Figure 8. As expected, elementary school-age children drive the effects on child maltreatment reports. The results indicate that 
child maltreatment reports for elementary school-age children decrease by $23 \%$ after schools closed, while the estimates for preschool are imprecisely estimated and for middle school are close to zero and statistically insignificant, offering support to our argument that teachers and school professionals are key in detecting and reporting child maltreatment.

Figure 8. Heterogeneous Effects of COVID-19 School Closures on Child Maltreatment by Age Group

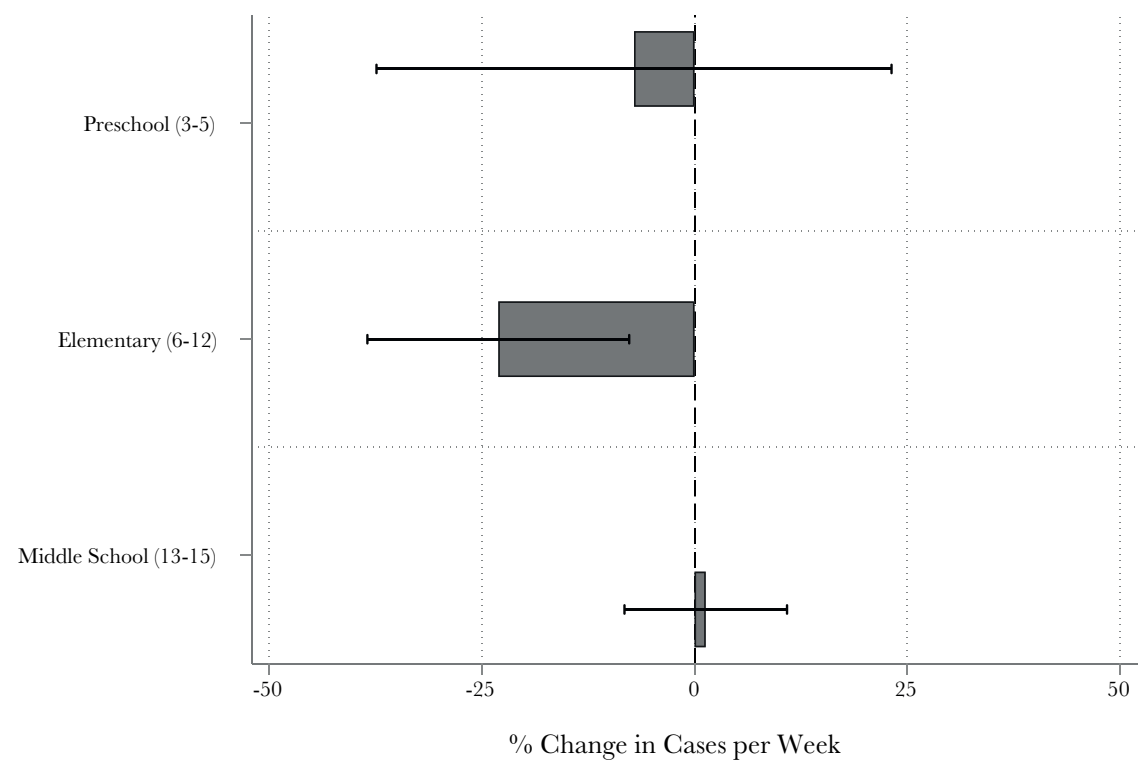

Notes: Each bar represents a different regression. All estimations include municipality, gender, week-year, municipality-week, and municipality-year fixed effects as well as municipality-specific and gender-specific linear time trends. Standard errors for 95\% confidence intervals are clustered at the municipality-by-gender level.

\section{Discussion of Results}

This paper provides early evidence on the effects of COVID-19 school closures on the reporting of child maltreatment. Estimates from synthetic control and difference-in-differences methods, respectively, show that child maltreatment reports decreased by 29 and 30 percent after schools closed on March 23, 2020. We argue that this decline is due to a reduction in reports by education professionals. The estimated decline in maltreatment reports is larger for women and in poorer municipalities.

These results are in line with recent evidence suggesting that poor economic conditions and COVID-19 school closures are related to lower reported violence against children (Stephens-Davidowitz, 2013; Baron et al., 2020), and contribute to the scarce evidence highlighting the importance of education professionals on detecting, counseling, and reporting violence on children that otherwise would have been missed (Fitzpatrick et al., 2020). Our results in a highly unequal setting also shed light on the importance of third-party adults on the reporting of child maltreatment to the authorities, the problem of under-detection, and how education professionals could be key in reporting maltreatment in more vulnerable settings and for girls. Finally, our heterogeneous effects showing that under-reporting in the context of school closures is higher for females, suggest the normalization of violence towards women at home and the need for policies aiming to detect and reduce such maltreatment.

Our heterogeneous results coupled with the literature showing long-term detrimental consequences on children's future labor and education outcomes suggest that under-reporting have the potential to exacerbate gender inequality in the future. These results call for policies aimed at identifying, preventing, and treating victims of child abuse, especially girls, to mitigate the negative consequences of school closures during the COVID-19 pandemic.

Finally, although estimating the effects of school closures, and more broadly of the COVID-19 pandemic, on the number of actual cases of child maltreatment on and the consequences of under-reporting, is outside the scope of this paper, understanding how the COVID-19 pandemic has 
F. Cabrera and M. Padilla

impacted the number of cases of child maltreatment, remains an important question for future research.

\section{Acknowledgments}

We thank Emily Pratt and Antonio Ruiz-Porras; seminar participants at Universidad del Norte, Universidad de Guadalajara, and IZA \& Jacobs Center Workshop: Consequences of COVID-19 for Child and Youth Development; and colleagues at the Center for Institutional Studies, HSE, Moscow, for helpful feedback on earlier versions of this paper.

\section{References}

Abadie, A., Diamond, A., \& Hainmueller, J. (2010). Synthetic control methods for comparative case studies: Estimating the effect of California's tobacco control program. Fournal of the American statistical Association, 105(490), 493-505.

Agüero, J. M. (2020). COVID-19 and the rise of intimate partner violence. World development, 137, 105-217.

Baron, E. J., Goldstein, E. G., \& Wallace, C. T. (2020). Suffering in silence: How COVID-19 school closures inhibit the reporting of child maltreatment. Available at SSRN 3601399.

Beland, L.-P., Brodeur, A., Haddad, J., \& Mikola, D. (2020). COVID-19, family stress and domestic violence: Remote work, isolation, and bargaining power (Tech. Rep.). GLO Discussion Paper.

Boserup, B., McKenney, M., \& Elkbuli, A. (2020). Alarming trends in US domestic violence during the COVID-19 pandemic. The American Fournal of Emergency Medicine, 38(12), 27532755 .

Carrell, S. E., Hoekstra, M., \& Kuka, E. (2018). The long-run effects of disruptive peers. American Economic Review, 108(11), 3377-3415.

Correia, S., Guimaraes, P., \& Zylkin, T. (2020). Fast Poisson estimation with high-dimensional fixed-effects. The Stata Fournal, 20(1), 95-115.

Cunradi, C. B., Mair, C., Ponicki, W., \& Remer, L. (2011). Alcohol outlets, neighborhood characteristics, and intimate partner violence: ecological analysis of a California city. Fournal of Urban Health, 88(2), 191-200.

Currie, J., \& Spatz Widom, C. (2010). Long-term consequences of child abuse and neglect on adult economic well-being. Child maltreatment, 15(2), 111-120.

Currie, J., \& Tekin, E. (2012). Understanding the cycle childhood maltreatment and future crime. Fournal of Human Resources, 47(2), 509-549.

Fitzpatrick, M. D., Benson, C., \& Bondurant, S. R. (2020). Beyond reading, writing, and arithmetic: The role of teachers and schools in reporting child maltreatment (Tech. Rep.). National Bureau of Economic Research.

Leslie, E., \& Wilson, R. (2020). Sheltering in place and domestic violence: Evidence from calls for service during COVID-19. Available at SSRN 3600646.

Lindo, J. M., Schaller, J., \& Hansen, B. (2018). Caution! Men not at work: Gender-specific labor market conditions and child maltreatment. Fournal of Public Economics, 163, 77-98.

Peterman, A., Potts, A., O’Donnell, M., Thompson, K., Shah, N., Oertelt-Prigione, S., \& van Gelder, N. (2020). Pandemics and violence against women and children (Working Paper No. 528). Center for Global Development. 
Ravindran, S., \& Shah, M. (2020). Unintended consequences of lockdowns: COVID-19 and the shadow pandemic (Tech. Rep.). National Bureau of Economic Research.

Silverio-Murillo, A., \& de la Miyar, J. R. B. (2020). Families under confinement: COVID-19, domestic violence, and alcohol consumption (Working Paper Series No. Forthcoming). Andrew Young School of Policy Studies.

Stephens-Davidowitz, S. (2013). Unreported victims of an economic downturn. (Unpublished Manuscript. Retrieved from: https://static1.squarespace.com/static/51d894bee4b01caf88ccb4f3/t/51e22f38e4b0502fe211fab7/1373777720363/childabusepaper13.pdf)

Vanderminden, J., Hamby, S., David-Ferdon, C., Kacha-Ochana, A., Merrick, M., Simon, T. R., Finkelhor, D., Turner, H. (2019). Rates of neglect in a national sample: Child and family characteristics and psychological impact. Child abuse \& neglect, 88, 256-265.

Zielinski, D. S. (2009). Child maltreatment and adult socioeconomic well-being. Child abuse \&尺 neglect, 33(10), 666-678.

Table A1. Number of Reports by Group of Age and their Incidence in Females and in Richer Municipalities

\begin{tabular}{rcccc}
\hline (1) Age & (2) Weeks & (3) Reports & $(4) \%$ Females & (5) \% Richer \\
\hline $3-15$ & 74 & 2727 & 50.71 & 23.43 \\
$16-21$ & 74 & 2774 & 85.69 & 19.07 \\
$22-27$ & 74 & 5007 & 89.22 & 18.05 \\
$28-33$ & 74 & 5138 & 86.84 & 21.39 \\
$34-39$ & 74 & 4370 & 84.79 & 21.98 \\
$40-45$ & 74 & 3561 & 80.43 & 22.43 \\
$46-51$ & 74 & 2864 & 75.85 & 22.94
\end{tabular}

Notes: Columns (4) and (5) show the percentage of reports in which the victim is a female, or that happen in the four "richest" municipalities or with highest Socio Development Index. 